\title{
Vehicle-to-Vehicle Channel Characterization Based on Ray- Tracing for Urban Road Scenarios
}

\author{
Lei Xiong $\mathbb{D}$, Zhiyi Yao $\mathbb{D}$, Haiyang Miao $\mathbb{D}$, and Bo Ai $\mathbb{D}$ \\ The State Key Laboratory of Rail Traffic Control and Safety, Beijing Jiaotong University, 100044 Beijing, China \\ Correspondence should be addressed to Lei Xiong; lxiong@bjtu.edu.cn
}

Received 28 August 2020; Revised 3 October 2020; Accepted 17 March 2021; Published 31 March 2021

Academic Editor: Alessandro Bazzi

Copyright ( 2021 Lei Xiong et al. This is an open access article distributed under the Creative Commons Attribution License, which permits unrestricted use, distribution, and reproduction in any medium, provided the original work is properly cited.

\begin{abstract}
In this paper, the vehicle-to-vehicle (V2V) channel characteristics in peak hours at the $5.9 \mathrm{GHz}$ band in two typical urban road scenarios, the urban straight road and the intersection, are investigated. The channel characteristics, such as path loss, root mean square (RMS) delay spread, and angular spread, are derived from the ray-tracing (RT) simulations. Due to the low height of antennas at both the transmitter (Tx) and the receiver (Rx), the line of sight (LOS) between the Tx and the Rx will often be obstructed by other vehicles. Based on the RT simulation results, the shadowing loss is modelled by the multimodal Gaussian distribution, and path loss models in both LOS and non-LOS (NLOS) conditions are obtained. And the RMS delay spread in two scenarios can be modelled by the Weibull distribution. In addition, the deployment of an antenna array is discussed based on the statistics distribution of the angular spread.
\end{abstract}

\section{Introduction}

As one of the important parts of an intelligent transportation system (ITS), the V2V communication system allows vehicles to exchange information about the surrounding traffic situation to improve safety, reduce traffic congestion, and provide a comfortable driving experience [1]. It is estimated by the U.S. Department of Transportation (DOT) that V2V communication can address up to $82 \%$ of all crashes, saving thousands of lives and billions of dollars. In recent years, $\mathrm{V} 2 \mathrm{~V}$ communication has attracted more and more attention and has achieved remarkable development.

The channel characteristics are of vital importance in the research, design, and deployment of the V2V communication system. Due to the low antenna height at both the Tx and the $\mathrm{Rx}$, rapid time-varying environments, high mobility of vehicles, relatively short communication distance, diversity of scattering objects, etc., the V2V channel characteristics are significantly different from those in the cellular networks [2].

Many measurement campaigns have been conducted to investigate the $\mathrm{V} 2 \mathrm{~V}$ channel characteristics over the past few years, and a number of $\mathrm{V} 2 \mathrm{~V}$ channel models have been proposed. Most campaigns are carried out between the vehicles on the straight road, either in the same direction [3-6] or in opposite [7, 8], and at intersections [9-11]. RT simulation technology is also used in [12], where the V2V channel characteristics of an urban intersection environment are analyzed, especially the power delay profile (PDP) and channel gain.

Many studies are carried out in LOS conditions, but some in NLOS conditions caused by large-size vehicles, such as buses, trucks, and vans. In [13], the shadowing effect caused by obstructing vehicles in the $\mathrm{V} 2 \mathrm{~V}$ channel has been addressed. In [14-16], based on the measurement in highways and urban environments, the shadow fading models 


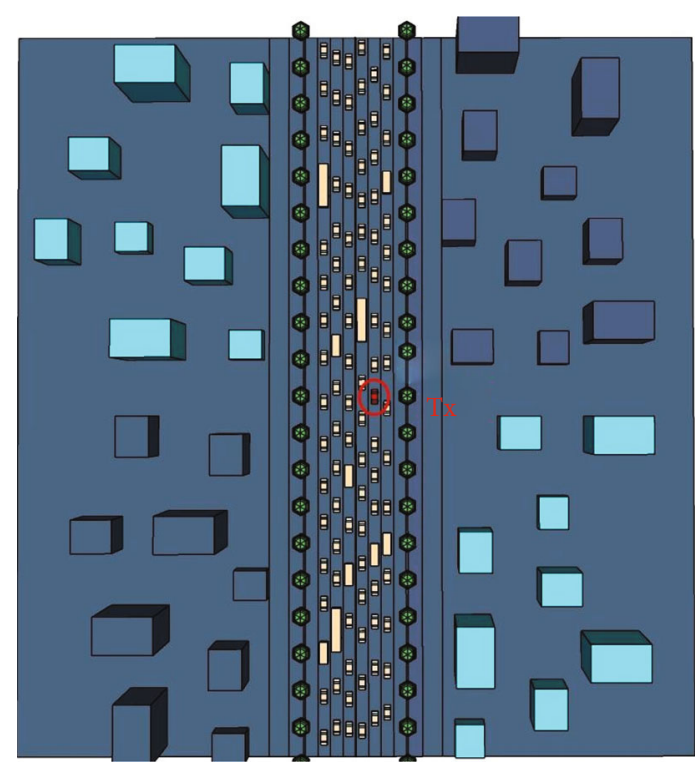

(a)

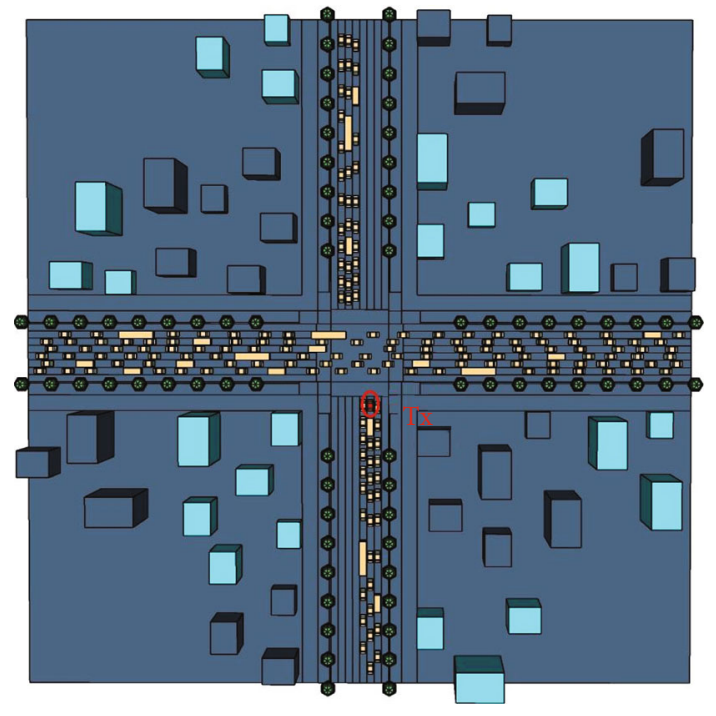

(b)

FIGURE 1: Straight road scenario and intersection scenario: (a) straight road; (b) intersection.

in LOS and NLOS conditions are established, and it is emphasized that the shadowing loss caused by the surrounding vehicles is about $10 \mathrm{~dB}$. In [17], the $\mathrm{V} 2 \mathrm{~V}$ communication link is divided into three categories: LOS, LOS blocked by vehicles, and LOS blocked by stationary objects, in which the obstructing vehicles have a more significant impact on the V2V channel. In [14], according to the causes of LOS blockage (vehicles or buildings), the NLOS regions are divided into obstructed LOS (OLOS) and NLOS with lognormal distribution, respectively.

$\mathrm{V} 2 \mathrm{~V}$ channel characteristics are related to the environment (i.e., urban, suburban, highway, etc.), vehicle speed, and traffic density. During peak hours, urban traffic densities are usually higher and vehicle speeds are usually lower. Due to the mobility of Tx/Rx, the LOS will be blocked and appear in NLOS conditions, which means it is more urgent to investigate the channel characteristics in peak hours. Therefore, in this paper, the $\mathrm{V} 2 \mathrm{~V}$ channel characteristics of an urban straight road and intersection, e.g., path loss, RMS delay spread, and angular spread, in peak hours at the $5.9 \mathrm{GHz}$ band are investigated based on RT. Channel characteristics in both LOS and NLOS conditions, the probability distribution of LOS in each scenario, and the distribution of shadowing loss and RMS delay spread are modelled. Finally, the RMS angular spread and antenna array deployment are discussed.

The rest of this paper is organized as follows. Section 2 presents the scenario models in urban roads and introduces the RT simulation technology. Section 3 investigates the channel characteristics in both LOS and NLOS conditions. It also includes the probability distribution of LOS in each scenario and the derivation of path loss and modeling of shadow fading in LOS and NLOS conditions. The deployment of an antenna array is also discussed. Conclusions are drawn in Section 4.

\section{Urban Road Scenario and Ray- Tracing Simulation}

2.1. Urban Road Modeling. The two typical V2V scenarios, urban straight road and intersection, are modelled in the paper. Multiple samples are established in these two scenarios, and Figure 1 takes one sample as an example in each scenario.

2.1.1. Straight Road. Six motor vehicle lanes and two nonmotor vehicle lanes are included. During peak hours, the speed of vehicles is relatively low $(25 \mathrm{~km} / \mathrm{h})$, and the distance between adjacent vehicles is uniformly distributed between 4 and $10 \mathrm{~m}$, where the distance between adjacent vehicles is defined as the distance from the rear of the front vehicle to the head of the adjacent rear vehicle on the same motor vehicle lane.

2.1.2. Intersection. The total length of the queues for the vehicles waiting for traffic lights from south to north is $50 \mathrm{~m}$, and the distance between adjacent vehicles is uniformly distributed between 1 and $3 \mathrm{~m}$. While in the same direction, for vehicles that are 50 to $100 \mathrm{~m}$ from the zebra crossing and driving into the queues, the distance between adjacent vehicles is uniformly distributed between 4 and $10 \mathrm{~m}$. And the speed of the vehicles in the east-west direction is $25 \mathrm{~km} / \mathrm{h}$, and the distance between adjacent vehicles is uniformly distributed between 4 and $10 \mathrm{~m}$, too.

2.2. Object Modeling. The surrounding objects in the $\mathrm{V} 2 \mathrm{~V}$ scenarios that will affect the radio propagation are considered, such as vehicles, buildings, and trees.

2.2.1. Vehicle Modeling. The vehicles in urban roads are divided into three types in terms of size, small-sized vehicles, 


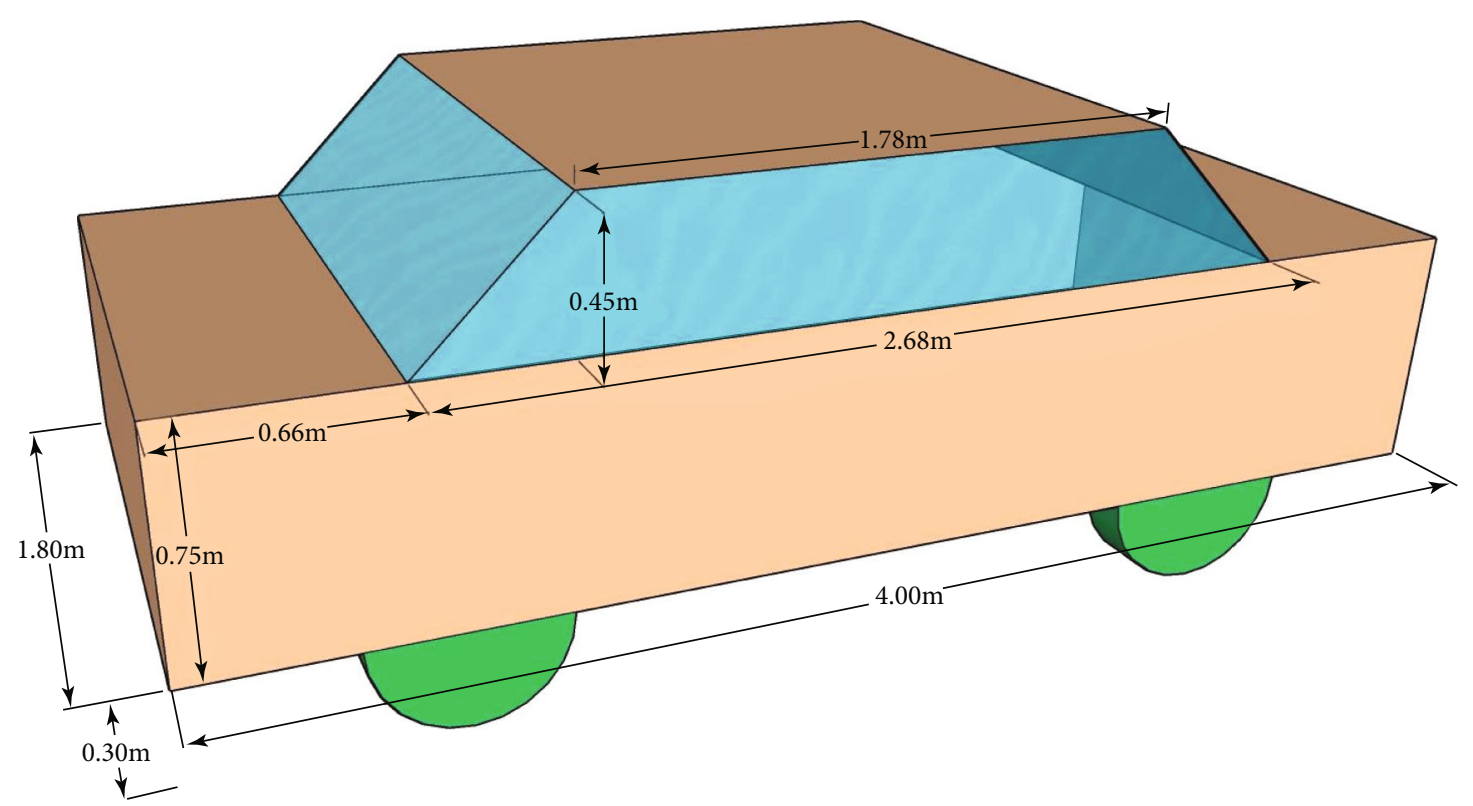

(a)

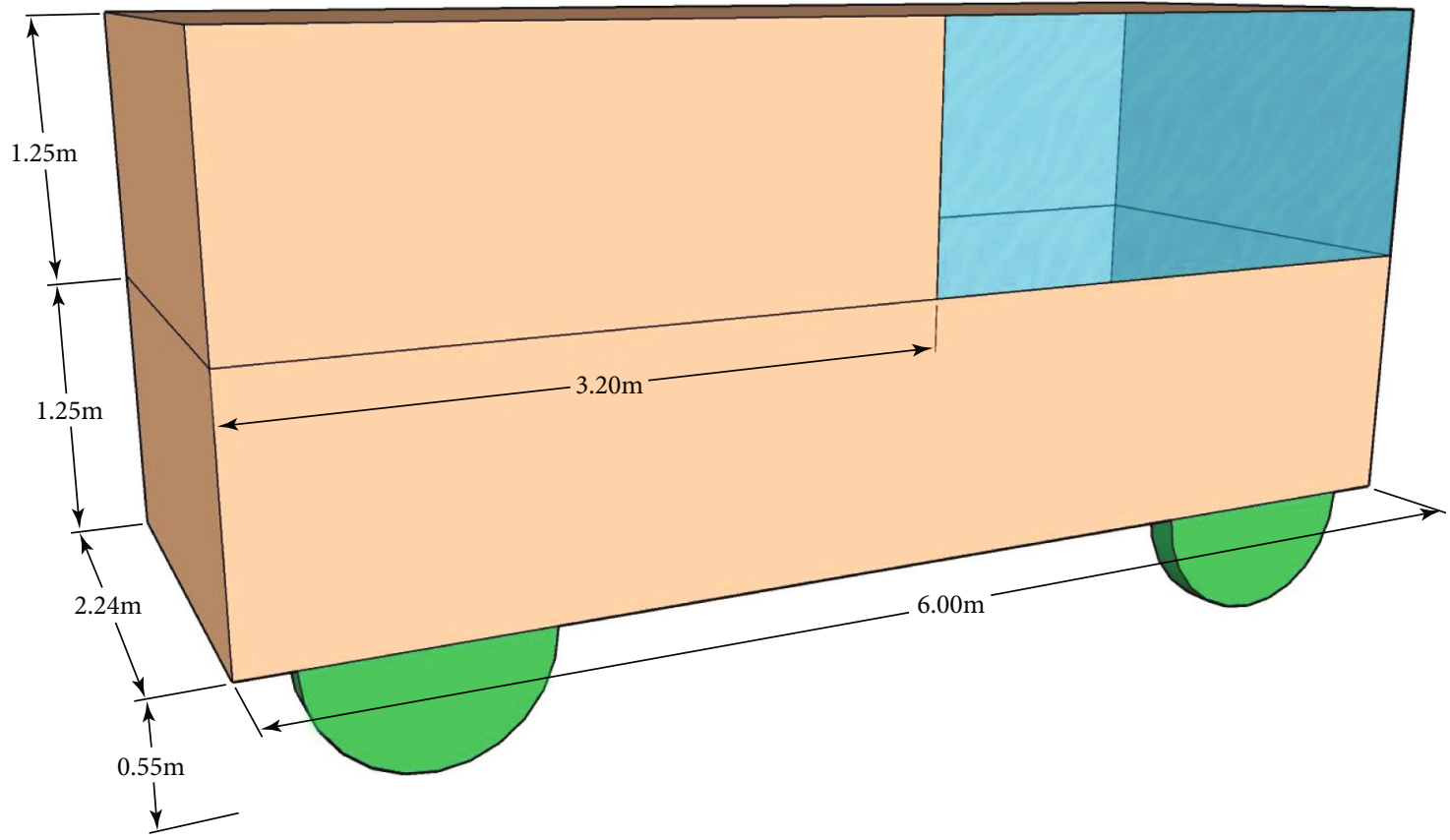

(b)

FIgure 2: Continued. 


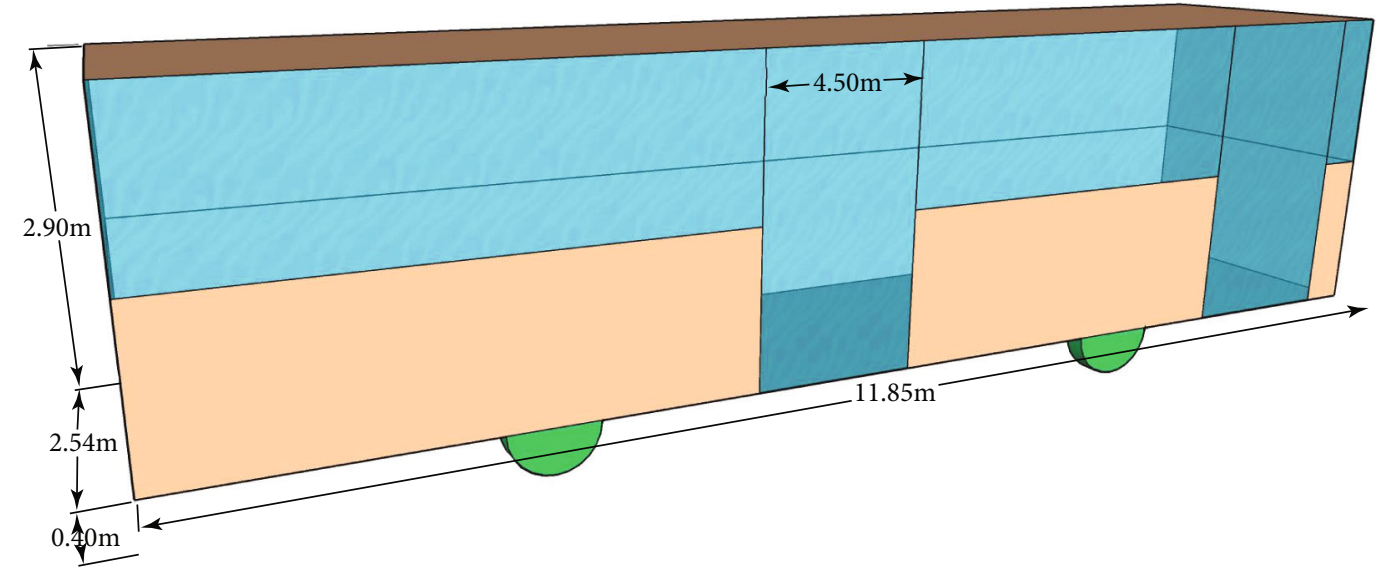

(c)

Figure 2: 3D model of vehicles: (a) car; (b) van; (c) bus.

medium-sized vehicles, and large vehicles, with the proportions of $89 \%, 7 \%$, and $4 \%$, respectively [18].

As shown in Figure 2, small-sized vehicles are modelled according to a 3-compartment car, medium-sized vehicles are modelled according to a van, and large-sized vehicles are modelled according to a bus.

2.2.2. Building Modeling. Urban buildings are modelled as low-rise, multistorey, and medium high-rise buildings, with the height of $7 \mathrm{~m}, 14 \mathrm{~m}$ and $23 \mathrm{~m}$ and the proportions of $20 \%, 50 \%$ and $30 \%$, respectively [19], as shown in Figure 3.

And the distribution of buildings presents a commercialresidential-commercial trend. The buildings are arranged with a spacing of $13 \mathrm{~m}$. The distance between a building and the sidewalk is $10 \mathrm{~m}$.

2.2.3. Tree Modeling. The trees are $0.5 \mathrm{~m}$ away from the edge of the road with an interval of $10 \mathrm{~m}$. The height of the trees is $10 \mathrm{~m}$, in which the heights of the trunk and the crown are both $5 \mathrm{~m}$, as shown in Figure 4 . The trunk is modelled as a hexagonal prism, while the crown is modelled as an approximate sphere.

In addition, the electromagnetic parameters of all the materials in the scenario are listed in Table 1.

2.3. Ray-Tracing Simulation. The $\mathrm{Tx} / \mathrm{Rx}$ antenna is placed on top of the car, with $0.05 \mathrm{~m}$ above the roof. For example, the omnidirectional antennas are used, as shown in Figure 5. And the simulation process is as follows.

2.3.1. Straight Road. A car in the middle lane is selected to simulate the V2V channel characteristics with other cars within 100 m, as shown in Figure 1(a).

2.3.2. Intersection. A car waiting for the traffic light near the zebra crossing in the north-south direction is selected, to simulate the V2V channel characteristics with other cars within around $100 \mathrm{~m}$, as shown in Figure 1(b).

As a technique to predict radio propagation characteristics, RT is based on electromagnetic theory, geometrical optics (GO) theory, and uniform theory of diffraction
(UTD). It is assumed that the radio wave propagates in the plane wave, whose far-field propagation characteristics can be simplified to a ray model to describe all propagation paths from $\mathrm{Tx}$ to $\mathrm{Rx}$, and the geometry and dielectric properties of scattering objects in the scenario need to be modelled.

The RT simulator employed in this study is developed by Beijing Jiaotong University. It is composed of a V2V RT simulator [20] and an UWB THz RT simulator [21]. Recently, this RT simulator is extended to a high-performance computing (HPC) cloud-based platform (CloudRT). More details of this platform can be found in [22] as well as via http:// raytracer.cloud. This $\mathrm{RT}$ simulator has been validated by a large number of measurements from frequencies below the $6 \mathrm{GHz}[1,20]$ to $300 \mathrm{GHz}$ band $[21,23]$. With a Web browser, MATLAB, and SketchUp installed, users can prepare the needed models, configure/trigger simulation tasks, and download results.

RT can identify all possible rays between $\mathrm{Tx}$ and $\mathrm{Rx}$, whose propagation modes include LOS, reflection, scattering, and diffraction. After a coherent superposition of all determined rays, the final output of the ray-based model is the time-variant $\operatorname{CIR} \mathbf{h}(\tau, t) \in \mathbb{C}^{M_{R} \cdot M_{T}}$, which completely characterizes the frequency-selective channel for each Tx/Rx link for the $M_{T}$ and $M_{R}$ transmit and receive antennas, respectively. We can express the CIR for single-input singleoutput (SISO) transmission, i.e., $M_{R}=M_{T}=1$, as

$$
h(\tau, t)=\sum_{k=1}^{N(t)} a_{k}(t) e^{j\left(2 \pi f \tau_{k}(t)+\varphi_{k}(t)\right)} \delta\left(\tau-\tau_{k}\right)=\sum_{k=1}^{N(t)} \tilde{a}_{k}(t) \delta\left(\tau-\tau_{k}\right),
$$

where the $k$ th MPC is described by the amplitude $a_{k}(t)$, the delay $\tau_{k}(t)$, and the phase shift $\varphi_{k}(t)$ at time $t . N(t)$ and $f$ denote the number of MPCs for each time instant and the carrier frequency, respectively. Based on the predicted CIR, additional characteristics like the PDP and RMS delay spread can be derived.

The simulation parameters are listed in Table 2, where the reflection order is defined as the maximum number of 

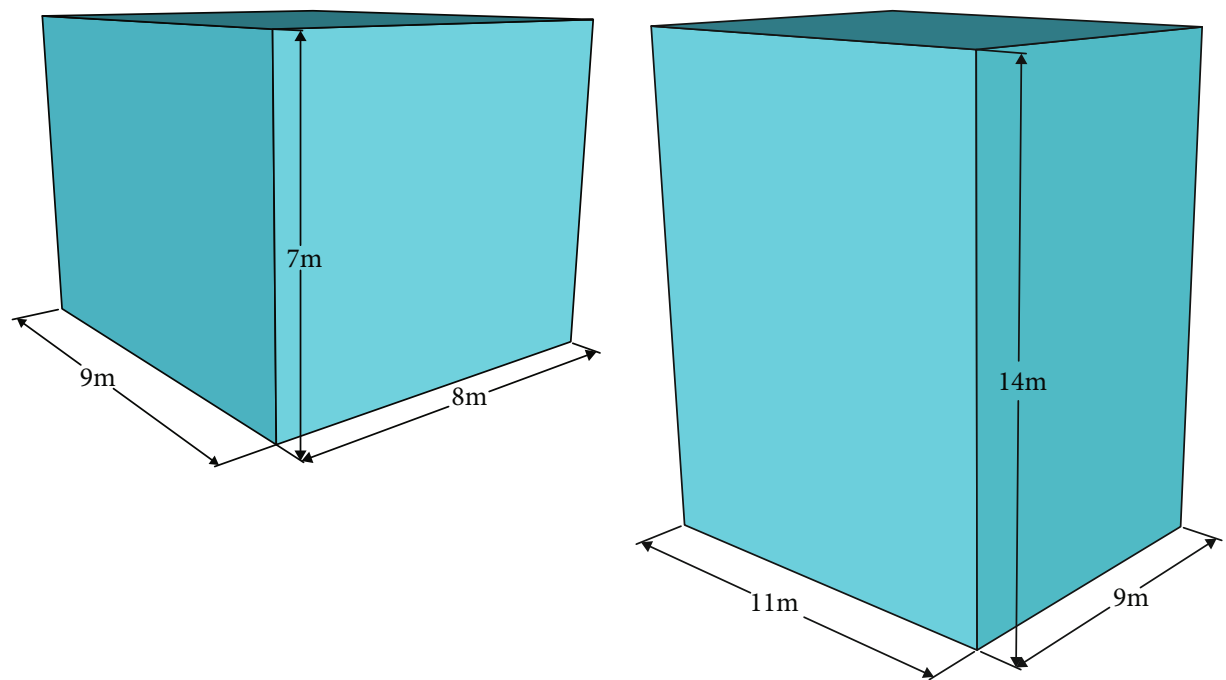

(a)

(b)

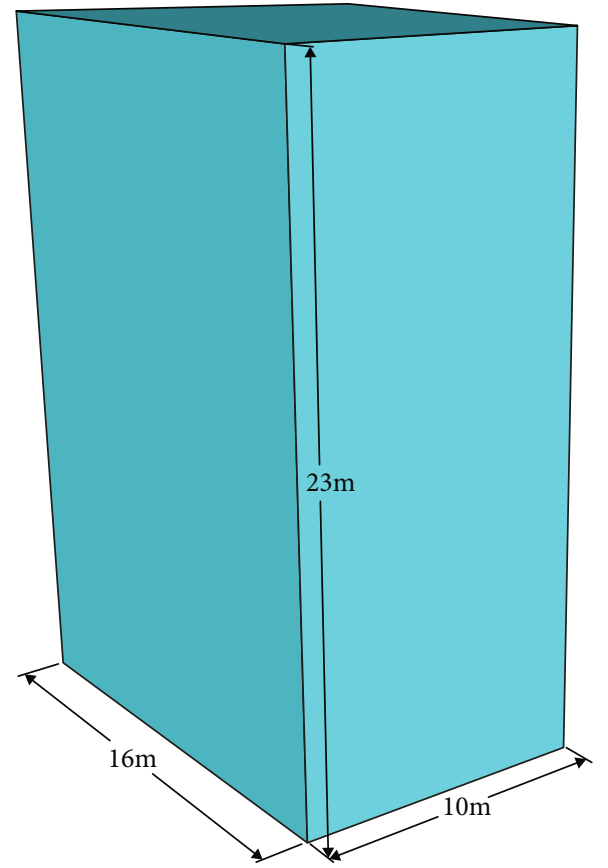

(c)

FIGURE 3: 3D model of buildings (take commercial buildings as an example): (a) low-rise buildings; (b) multistorey buildings; (c) medium high-rise buildings.

reflections that can be determined by the RT model based on the image method. Specular reflections are calculated recursively up to a desired order, but considering the complexity of the environment, only reflections up to order three are practical due to the limit of computational effort. In addition, the directive scattering (DS) model [24] which is widely used in optics is selected to calculate the power and direction of the scattering path in the platform.

\section{Channel Characteristics}

In this section, based on RT simulation technology, the channel characteristics in the straight road and intersection are discussed in peak hours.
3.1. LOS and NLOS. It can be seen from Figure 6(a), in the straight road scenario, as the Tx-Rx distance increases from $10 \mathrm{~m}$ to $90 \mathrm{~m}$, the LOS probability falls from $100 \%$ to $40 \%$.

As shown in Figure 6(b), the LOS probability in the intersection scenario has a similar trend. However, the LOS probability in different regions (from south to north, from north to south, and in east-west direction) is slightly different. For cars driving from north to south, if there are medium-sized or large-sized vehicles in the east-west direction in front of the Tx, LOS will be blocked. However, the distance between adjacent vehicles is relatively short in peak hours, which results in about 4-6 vehicles in the east-west direction ahead of the Tx. In addition, considering the proportion of vehicles (the probability of the appearance of medium-sized and 


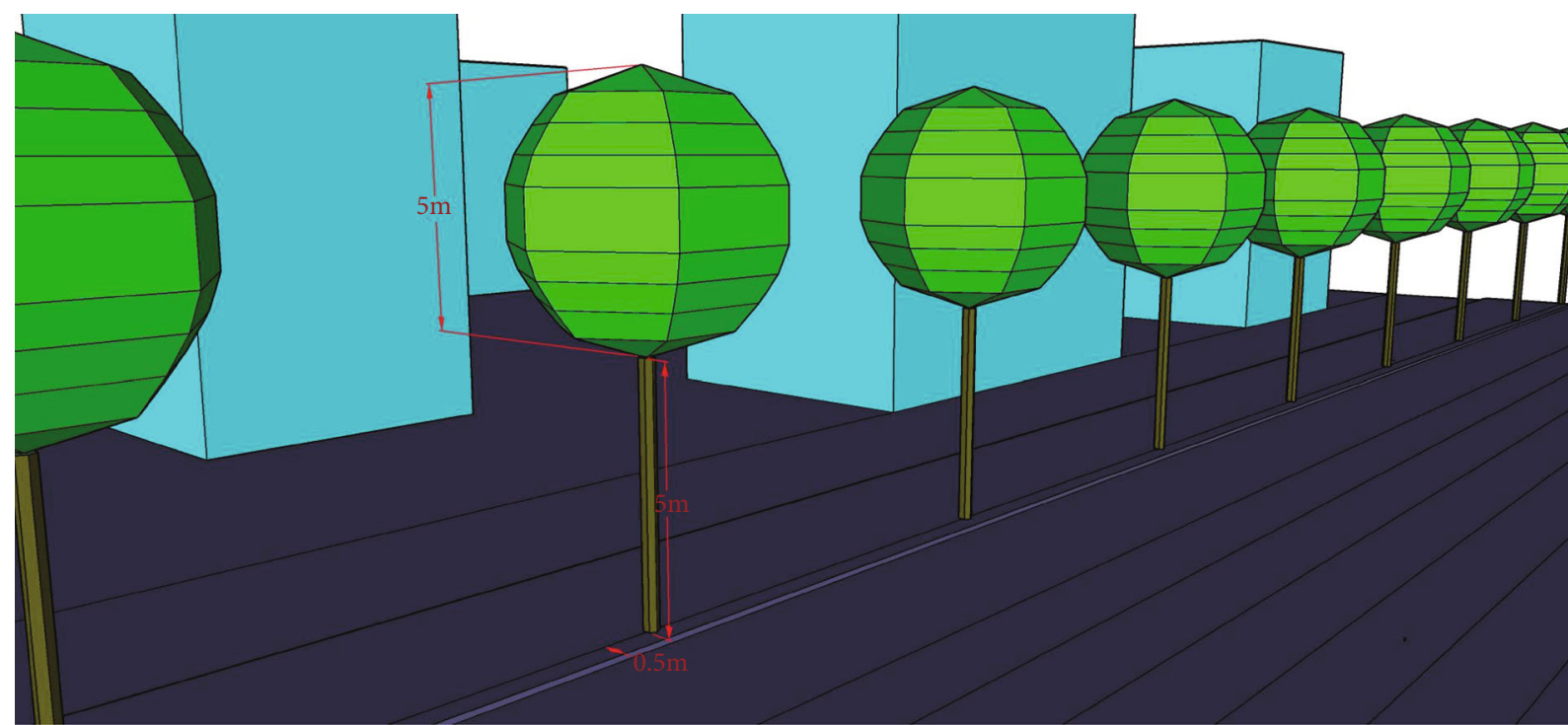

FIGURE 4: 3D model of trees.

TABLE 1: Material parameters.

\begin{tabular}{lccc}
\hline Object & Material & Relative permittivity & Loss tangent \\
\hline Ground, sidewalk, and residential buildings & Concrete & 1.06 & 0.65 \\
Vehicle windows, commercial buildings & Tempered glass & 0.0538 & 23.9211 \\
Trunk & Wood & 1.27 & 0.0038 \\
Vehicle body, fence & Metal & 1 & $10^{7}$ \\
Leaves & Matsuba & 1.36 & 0.0441 \\
Wheel & Rubber & 2.168 & 0.038 \\
\hline
\end{tabular}

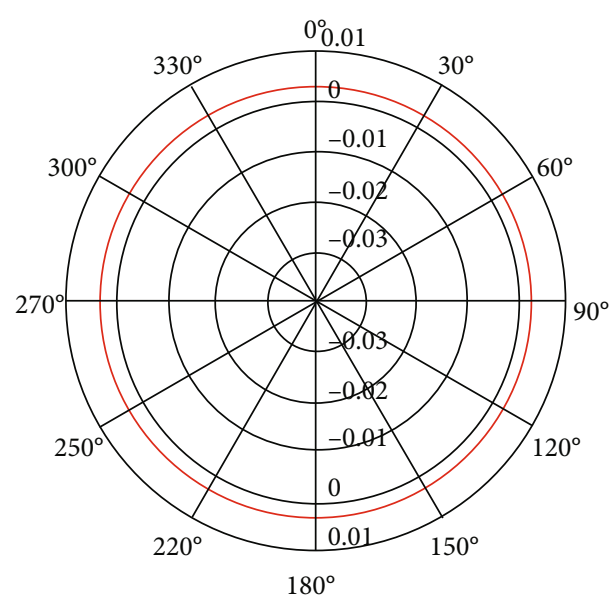

(a)

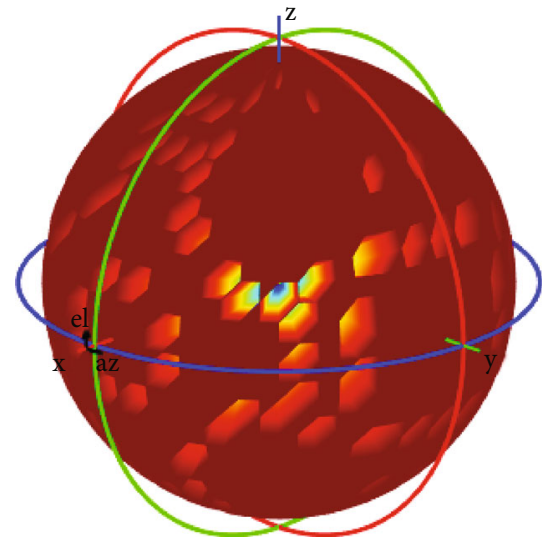

(b)

FIgURE 5: Omnidirectional antenna: (a) vertical cut; (b) 3D pattern.

TABLE 2: Simulation parameters.

large-sized vehicles appearing in urban roads is $11 \%$ ), according to probability theory, the probability of more than one large- and medium-sized vehicle in these 4-6 vehicles, that is, the probability of LOS blockage, will be more than $40 \%$, which means that the probability of LOS in that area will be less than $60 \%$.

\begin{tabular}{lc}
\hline Parameter & Value \\
\hline Frequency $(\mathrm{GHz})$ & $5.85-5.95$ \\
Resolution $(\mathrm{MHz})$ & 1 \\
Reflection order & 3 \\
Scattering mode & Directive mode \\
Antenna (Tx, Rx) & Omnidirectional vertical polarization \\
\hline
\end{tabular}




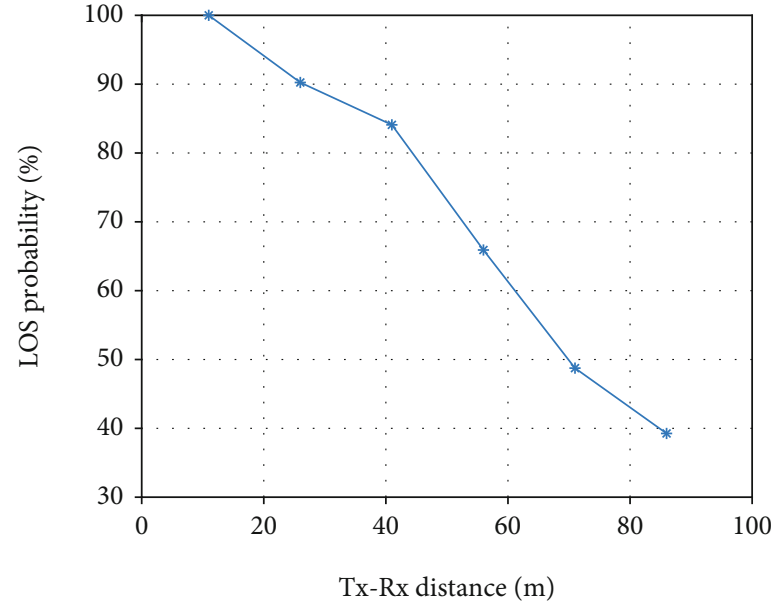

(a)

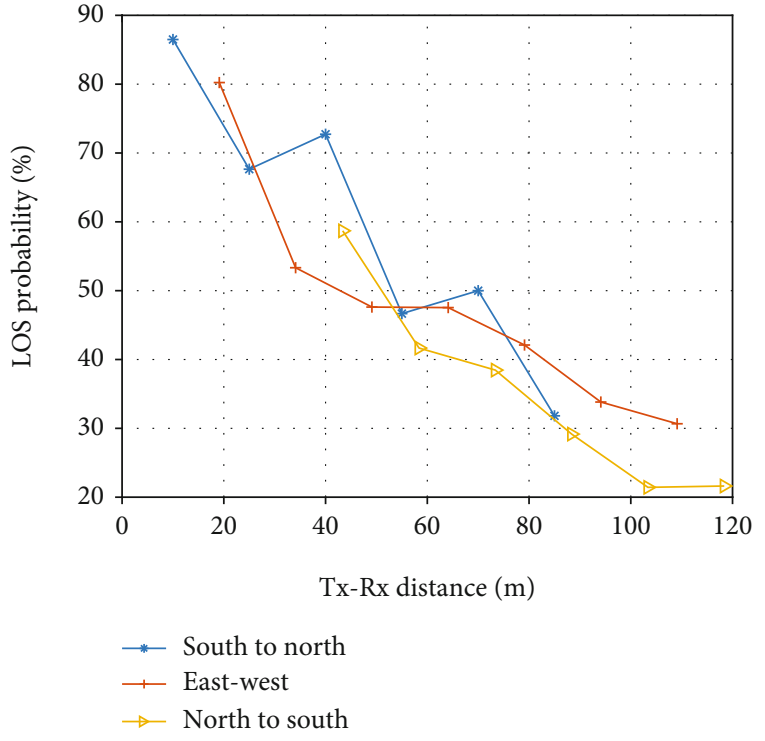

(b)

FIGURE 6: LOS probability at (a) straight road and (b) intersection.

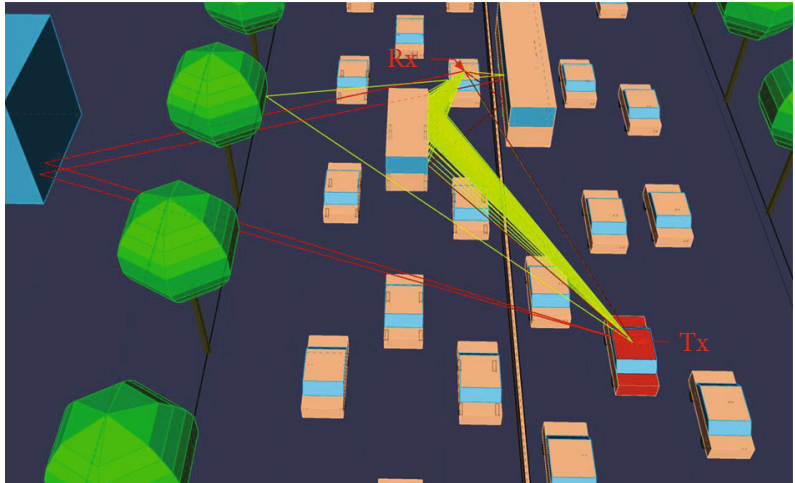

(a)

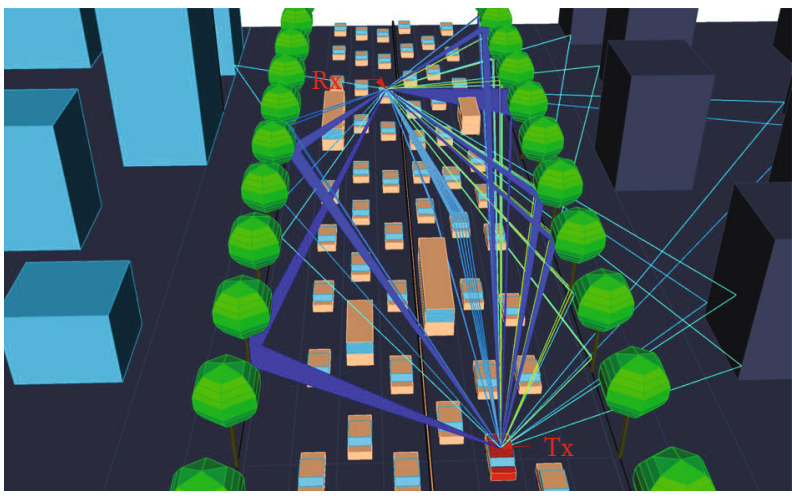

(c)

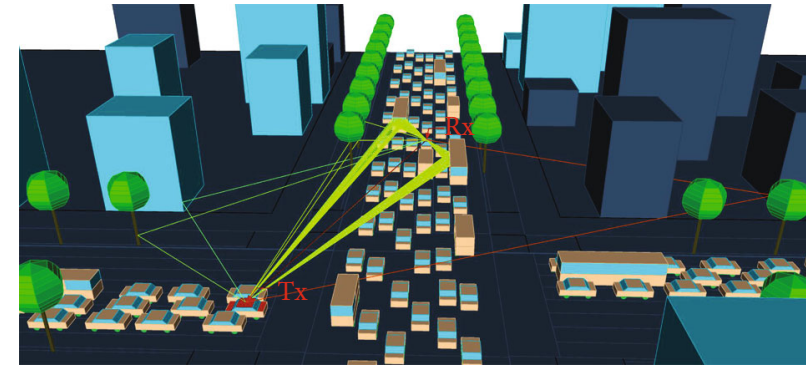

(b)

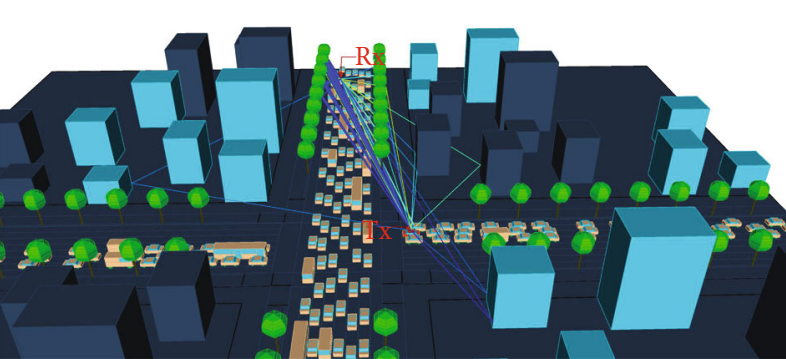

(d)

Figure 7: Propagation paths: (a) straight road scenario with LOS condition; (b) intersection scenario with LOS condition; (c) straight road scenario with NLOS condition; (d) intersection scenario with NLOS condition.

Figure 7 presents propagation paths in the straight road scenario and the intersection scenario in LOS and NLOS conditions.

In LOS conditions, there are a great number of reflections by the sides of the vehicle or the lower part of the side build- ing. In NLOS conditions, the reflection rays, mainly from the sides of vehicles and buildings, if they exist, will dominate over a large number of scattering rays with relatively low power caused by the vehicles, buildings, and trees. Moreover, 


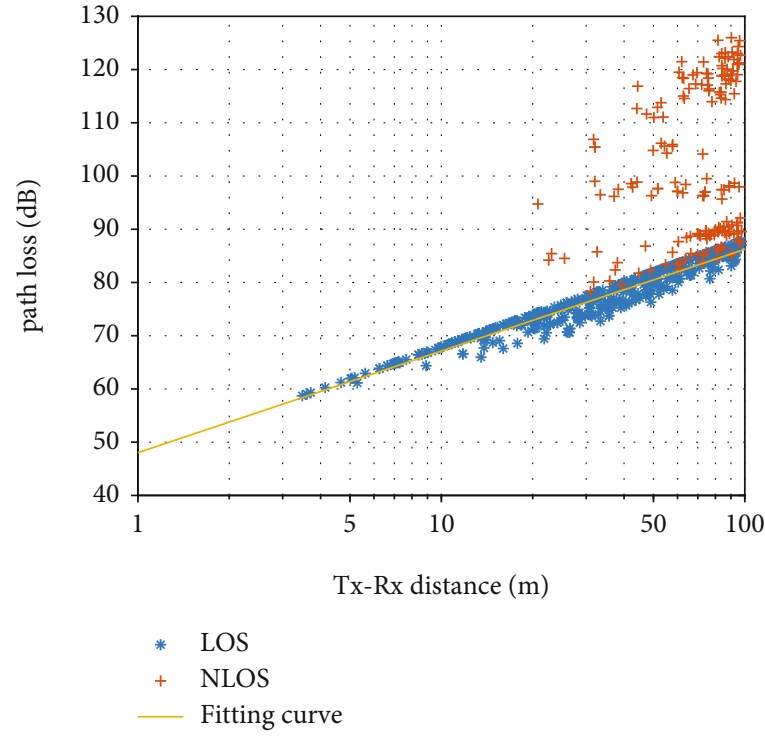

(a)

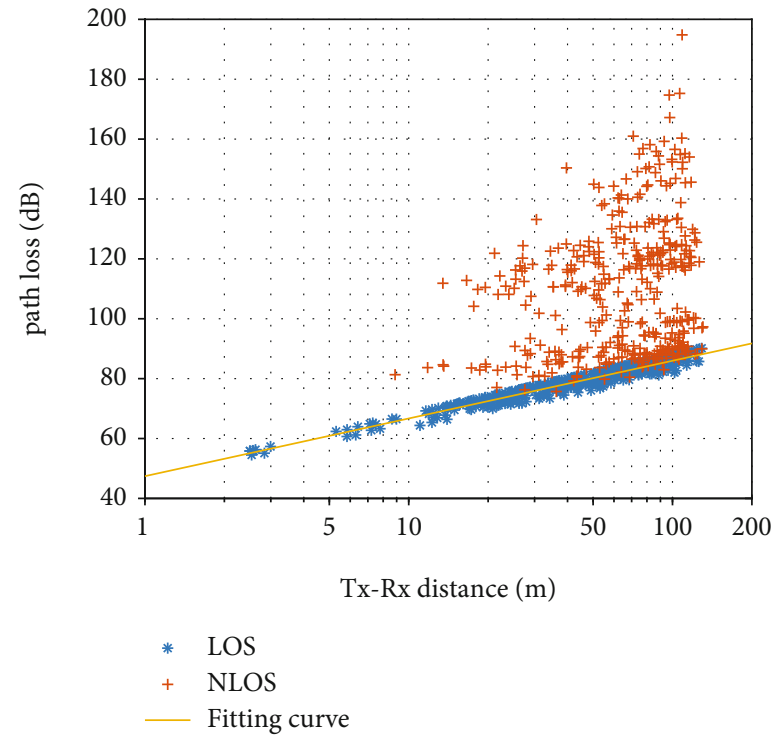

(b)

FIgURE 8: Path loss in terms of Tx-Rx distance at (a) straight road and (b) intersection.

TABLE 3: Parameters in path loss model.

\begin{tabular}{lcc}
\hline Scenario & Parameter & Value \\
\hline \multirow{2}{*}{ Straight road } & $n$ & 1.937 \\
& $A(\mathrm{~dB})$ & 47.55 \\
\hline \multirow{2}{*}{ Intersection } & $n$ & 1.927 \\
& $A(\mathrm{~dB})$ & 47.43 \\
\hline
\end{tabular}

it can be seen that more reflecting objects (i.e., buildings and trees) exist near the $\mathrm{Tx}$ and $\mathrm{Rx}$ in the intersection scenario, so more multipath contributions (MPCs) can be provided when the Tx and the Rx are obstructed. According to ITU-R P.1411 [25], the radio propagation within $1 \mathrm{~km}$ is mainly affected by buildings and trees. Since most of the short-range wireless signal transmission is in the city and suburb, the influence of buildings is particularly important. The height of transmitters is near ground level, which leads to more interaction with objects in the close neighbor surroundings [26].

3.2. Path Loss. Figure 8 shows the path loss in the straight road scenario and the intersection scenario with LOS and NLOS conditions in terms of the Tx-Rx distance. The path loss in $\mathrm{dB}$ is roughly linear with the logarithmic distance in LOS conditions. In NLOS conditions, the shadowing loss caused by vehicle occlusion can exceed $20 \mathrm{~dB}$ even in the short $\mathrm{Tx}-\mathrm{Rx}$ distance about $10 \mathrm{~m}$, which is consistent with the conclusion in [13].

Considering that NLOS conditions will have a great impact on the path loss, in order to improve the accuracy, the path loss model, shown in (2), is used in LOS and NLOS conditions, independently, and the fitting parameters are shown in Table 3.

$$
\mathrm{PL}(d)=A+10 n \log _{10}(d)+ \begin{cases}L_{a}, & \mathrm{LOS}, \\ L_{b}, & \text { NLOS }\end{cases}
$$

where $A$ is the interception, $d$ is the Tx-Rx distance (unit: $\mathrm{m}$ ), $n$ is the path loss exponent, and $L_{a}$ and $L_{b}$ are the shadowing loss in the LOS and NLOS conditions, respectively. Due to the obvious linear variation of the path loss in LOS conditions, both $n$ and $A$ are fitted in LOS conditions based on the minimum mean squared error (MMSE).

In the two scenarios, the waveguide effect occurs because of a set of waves reflected by the buildings on both sides of the road like valleys. Thus, the path loss exponent $n$ in the straight road and the intersection are slightly smaller than those in the free space path loss (FSPL) model $(n=2)$. Moreover, the phenomenon $(n<2)$ has also been found in many other studies [4, 27-29]. And the difference of $n$ and $A$ in the two scenarios is very small.

The probability density function (PDF) of the shadowing loss and fitting results in the straight road scenario and the intersection scenario with LOS conditions is shown in Figure 9. It should be noted that the change trend of simulated data is approximately monotonically increasing, which is similar to special cases of Weibull distribution. Reference [30] points out that the Weibull distribution is a better fit over all empirical data than the Nakagami distribution in V2V channels. The PDF of the Weibull distribution is defined as

$$
f(x, \lambda, k)=\frac{k}{\lambda}\left(\frac{x}{\lambda}\right)^{k-1} e^{-(x / \lambda)^{k}},
$$

where $\lambda>0$ denotes the scale parameter and $k>0$ denotes the shape parameter. And the cumulative distribution 


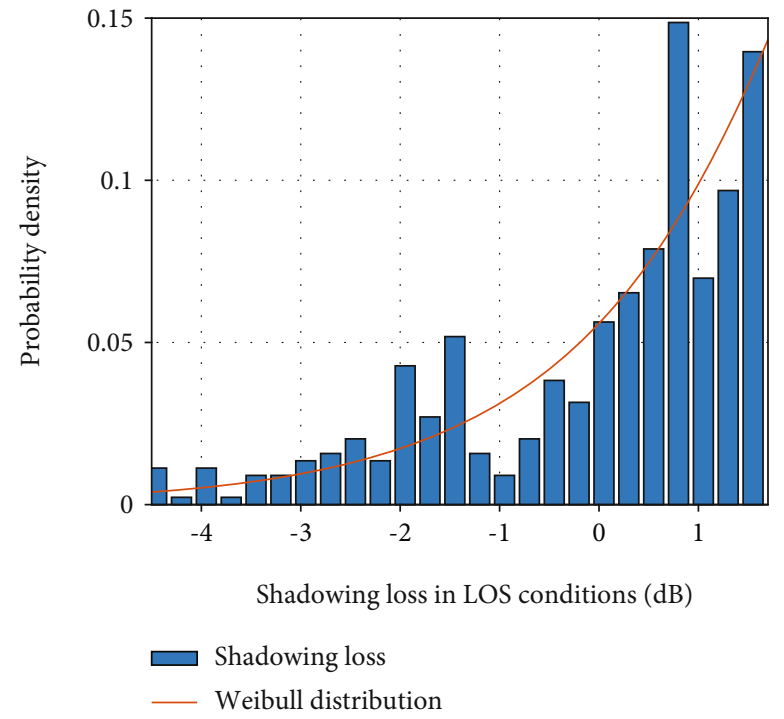

(a)

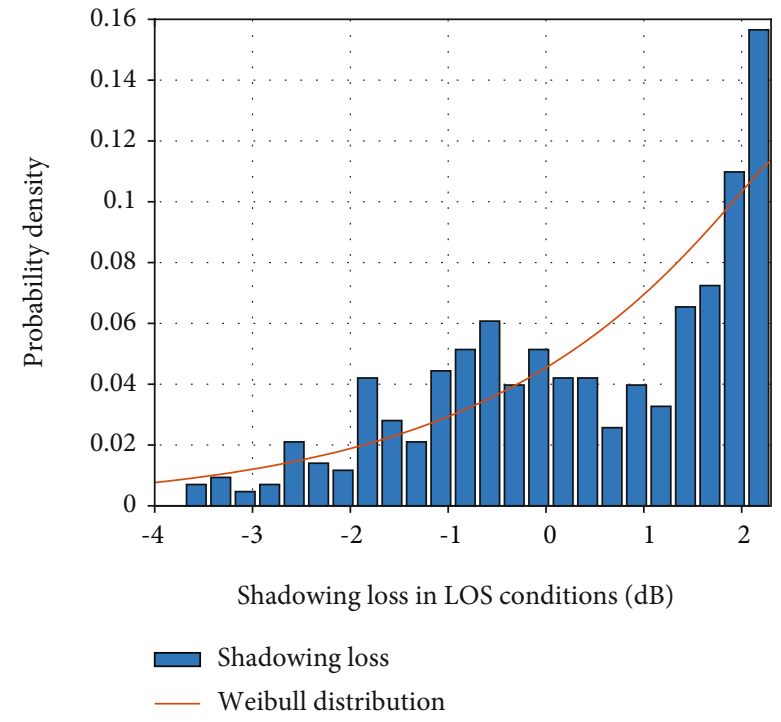

(b)

FIGURE 9: Shadowing loss distribution in LOS conditions: (a) straight road; (b) intersection.

TABLE 4: Parameters in shadowing loss model.

\begin{tabular}{lcc}
\hline Scenario & Straight road & Intersection \\
\hline$\lambda$ & 1.74 & 2.32 \\
$k$ & 1.02 & 1.03 \\
$u_{1}$ & 4.02 & 6.55 \\
$\sigma_{1}$ & 3.00 & 8.51 \\
$u_{2}$ & 16.94 & 37.04 \\
$\sigma_{2}$ & 6.32 & 10.2 \\
$u_{3}$ & 34.48 & 63.58 \\
$\sigma_{3}$ & 5.08 & 7.22 \\
$a$ & 0.60 & 0.46 \\
$b$ & 0.07 & 0.38 \\
\hline
\end{tabular}

function $(\mathrm{CDF})$ is defined by

$$
F(x, \lambda, k)=1-e^{-(x / \lambda)^{k}}
$$

In this paper, we need to modify the value of $x$ by substituting the value of the maximum shadowing loss minus the shadowing loss; the fitting parameters of the Weibull distribution are presented in Table 4 . The $\lambda$ in the intersection is larger than that in the straight road, which shows that the probability of different shadowing loss values in the intersection with LOS conditions is more uniform. In the intersection, although the influence of multipaths at different locations is diversified, the effect of MPCs is more consistent in the distribution of the shadowing loss. The differences in the shadowing loss in LOS conditions are mainly attributed to the different participations of multipaths on the direct LOS path.

The PDFs of the shadowing loss and fitting results in the straight road scenario and the intersection scenario with
NLOS conditions are shown in Figure 10. Observed from data fitting, it is more in line with the multimodal Gaussian distribution, which is the superposition of three Gaussian distributions, corresponding to different compositions of multipath components, and can well describe the statistics of the shadowing loss, whether there is a straight road or a crossroad. Fitting parameters of the shadowing loss are shown in Table 4. The multimodal Gaussian distribution is defined as

$$
\begin{aligned}
f\left(x, u_{1}, \sigma_{1}, u_{2}, \sigma_{2}, u_{3}, \sigma_{3}, a, b\right)= & a \frac{1}{\sqrt{2 \pi} \sigma_{1}} \exp \left(-\frac{\left(x-u_{1}\right)^{2}}{2 \sigma_{1}^{2}}\right) \\
& +b \frac{1}{\sqrt{2 \pi} \sigma_{2}} \exp \left(-\frac{\left(x-u_{2}\right)^{2}}{2 \sigma_{2}^{2}}\right) \\
& +(1-a-b) \frac{1}{\sqrt{2 \pi} \sigma_{3}} \exp \left(-\frac{\left(x-u_{3}\right)^{2}}{2 \sigma_{3}^{2}}\right),
\end{aligned}
$$

where $u_{1}, u_{2}$, and $u_{3}$ are the mean values of the Gaussian distribution from left to right and $\sigma_{1}, \sigma_{2}$, and $\sigma_{3}$ are the corresponding standard deviations.

The mean value of the first Gaussian distribution of shadowing loss is $4.02 \mathrm{~dB}$ (straight road) $/ 6.55 \mathrm{~dB}$ (intersection). This is mainly due to the existence of the single bounce reflections from the side of buildings and vehicles. Moreover, due to the larger value of $a$, the probability of the first Gaussian distribution is the largest. In [17,31], it is reported that, in the absence of LOS, most of the power is received by single bounce reflections from physical objects. The measurement results in the urban road in [14] at the $5.6 \mathrm{GHz}$ band show that the shadowing loss caused by obstructing vehicles follows the Gaussian distribution, and the mean value of the shadowing loss is about $7 \mathrm{~dB}$, which is basically consistent 


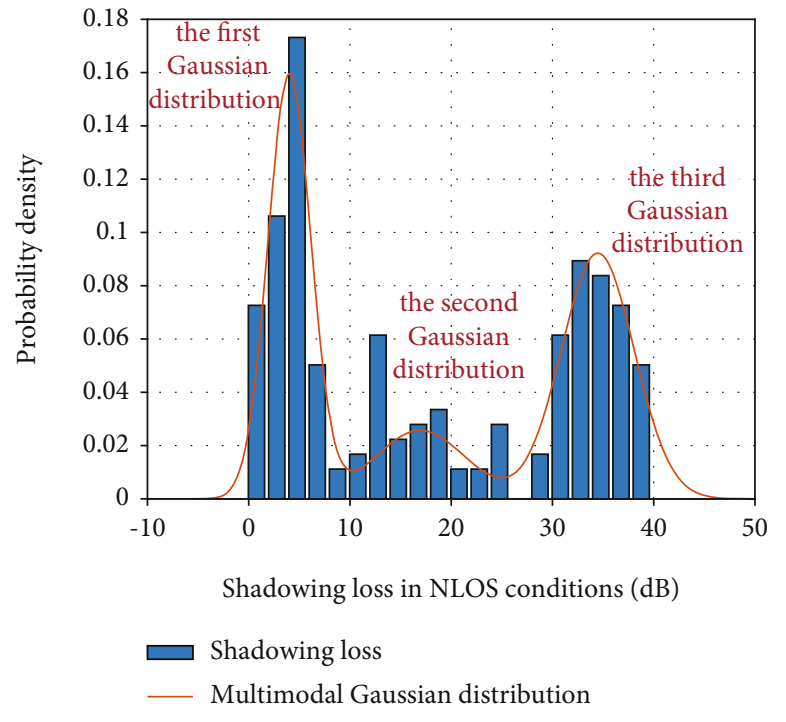

(a)

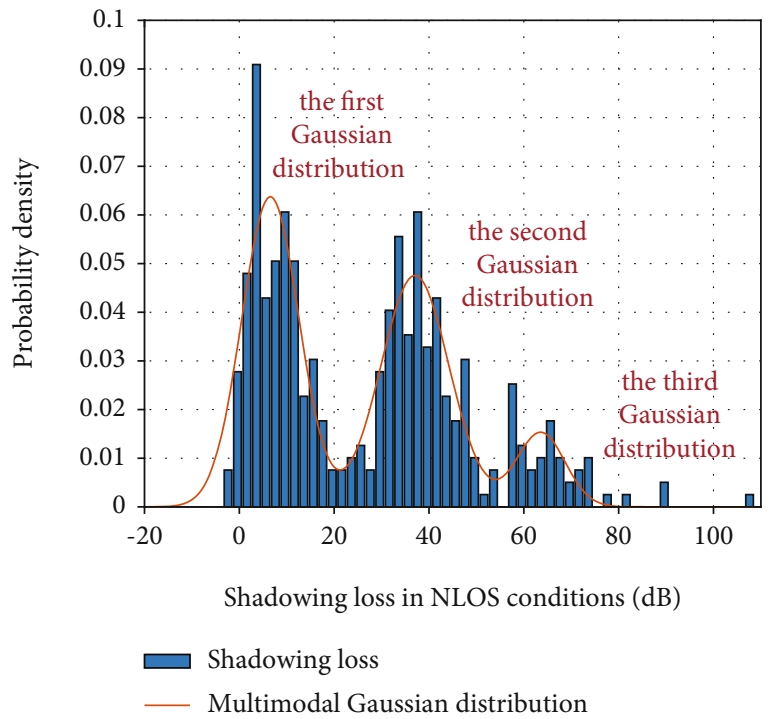

(b)

FIgURE 10: Shadowing loss distribution in NLOS conditions: (a) straight road; (b) intersection.

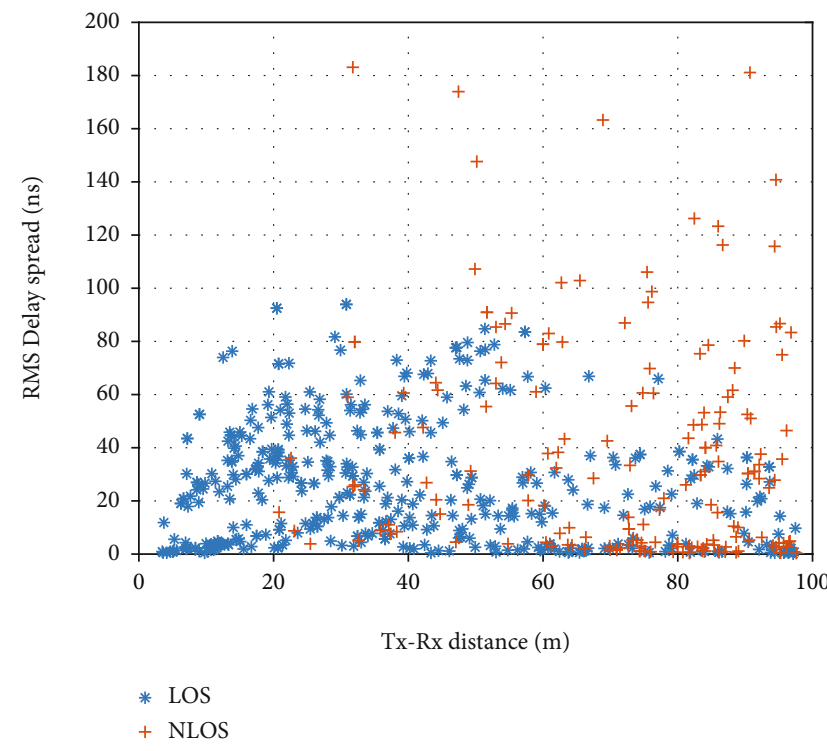

(a)

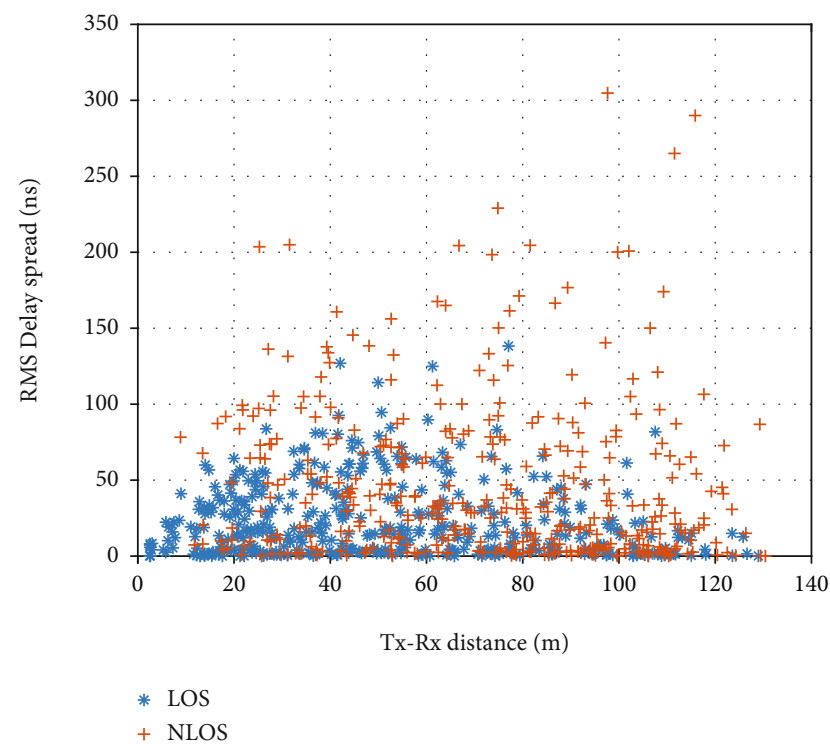

(b)

FIgURE 11: RMS delay spread in both LOS and NLOS conditions: (a) straight road; (b) intersection.

with the mean value of the first Gaussian distribution of the shadowing loss in this paper.

The mean value of the second Gaussian distribution of the shadowing loss in the straight road is $16.94 \mathrm{~dB}$, with a low probability. It is mainly caused by multiple reflections from the vehicles.

The mean value of the second Gaussian distribution of the shadowing loss in the intersection and the third Gaussian distribution of the shadowing loss in the straight road is
$34.48 \mathrm{~dB}$ (straight road)/37.06 dB (intersection). The rays are composed of a large number of scattering paths with a relatively low power from trees, buildings, and vehicles.

The mean value of the third Gaussian distribution of the shadowing loss in the intersection is $63.6 \mathrm{~dB}$. It only appears when the $\mathrm{Rx}$ is driving in the east-west direction, extremely far away from the intersection and obstructed by many medium-sized or large-sized vehicles, resulting in only a small number of scattering paths existing, with lower power. 


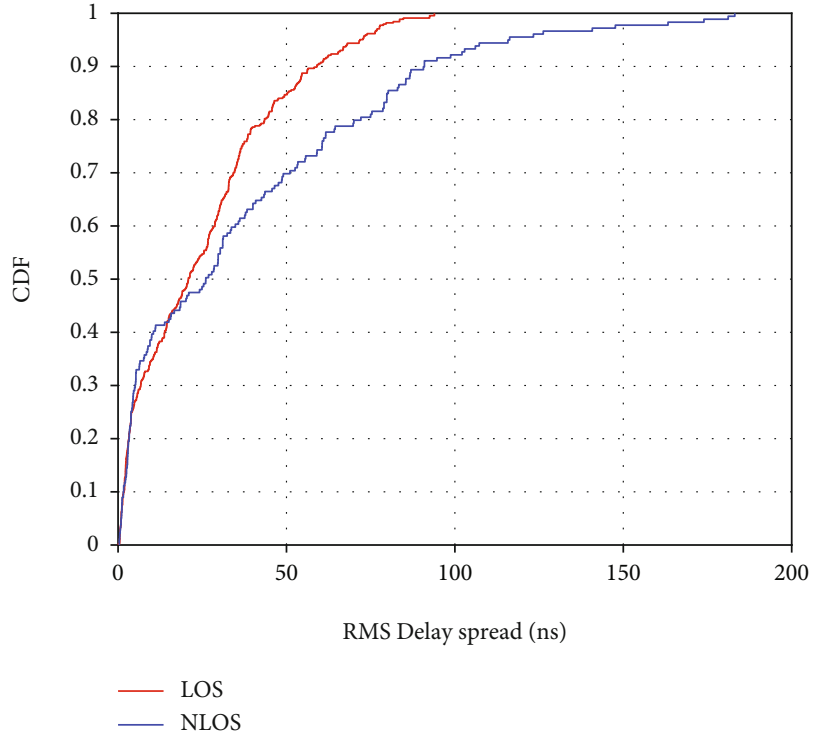

(a)

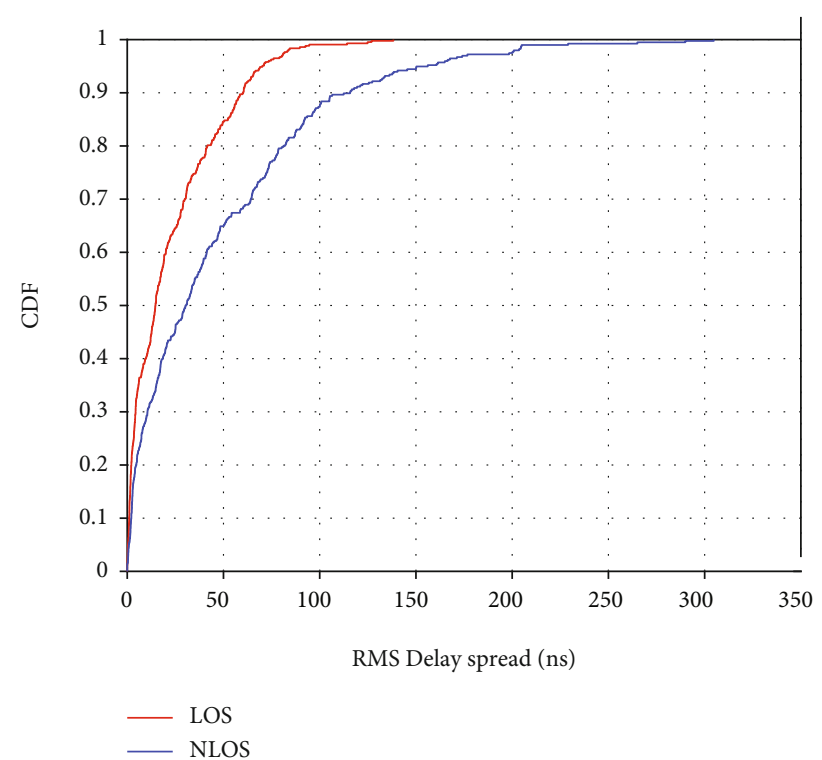

(b)

FIGURE 12: CDFs of RMS delay spread in both LOS and NLOS conditions: (a) straight road; (b) intersection.

Reference [9] points out that the path loss is high when the vehicles are far away from the intersection, with few physical objects actually providing propagation rays. In NLOS conditions, the strength of the received power is dependent on the availability of the reflection and scattering coming from buildings and trees, which may account for the main received power. In the absence of a large fraction of these reflections and scattering, the second Gaussian distribution or the third Gaussian distribution of the shadowing loss will appear.

3.3. RMS Delay Spread. The RMS delay spread is defined as [32]

$$
\tau_{\mathrm{rms}}=\sqrt{\frac{\sum_{n=1}^{N} P_{n}\left(\tau_{n}-\bar{\tau}\right)^{2}}{\sum_{n=1}^{N} P_{n}}},
$$

where $P_{n}$ and $\tau_{n}$ denote the power and the excess delay of the $n$-th ray, respectively; $N$ is the number of rays; and the mean delay $\bar{\tau}$ is defined by

$$
\bar{\tau}=\frac{\sum_{n=1}^{N} P_{n} \tau_{n}}{\sum_{n=1}^{N} P_{n}}
$$

Figure 11 shows the RMS delay spread in the straight road scenario and the intersection scenario with LOS and NLOS conditions. And the corresponding CDFs and PDFs are shown in Figures 12 and 13.

The median values of the RMS delay spread in the straight road are $20.86 \mathrm{~ns}$ (LOS) and $26.04 \mathrm{~ns}$ (NLOS), and $90 \%$ of the RMS delay spread are less than $58.24 \mathrm{~ns}$ (LOS) and $102.9 \mathrm{~ns}$ (NLOS). The median values of the RMS delay spread in the intersection are $20.86 \mathrm{~ns}$ (LOS) and $26.04 \mathrm{~ns}$ (NLOS), and $90 \%$ of the RMS delay spread are less than $58.24 \mathrm{~ns}$ (LOS) and $102.9 \mathrm{~ns}$ (NLOS).
The standard deviations of the RMS delay spread are $29.32 \mathrm{~ns}$ in the straight road and $41.66 \mathrm{~ns}$ in the intersection. The standard deviation in the intersection is larger than that in the straight road. The result illustrates that the surrounding scatterers in the intersection have a more significant impact on the V2V radio channel.

It can be observed that the RMS delay spread in LOS conditions is much smaller than that in NLOS conditions. The RMS delay spread in most locations is close to $0 \mathrm{~ns}$, which indicates that MPCs with a large delay suffer greater attenuation.

Moreover, the RMS delay spread in the intersection is larger than that in the straight road. MPCs will be involved, and the $\mathrm{Rx}$ can receive more reflection and scattering paths from the surrounding buildings and trees, even from some distant buildings, as shown in Figures 6(b) and 6(d), which leads to a greater RMS delay spread.

Generally, the statistical characteristics of the RMS delay spread can be modelled as a Gaussian distribution [33, 34]. However, as can be seen from Figure 12, the Gaussian distribution is not applicable, so we use the Weibull distribution to model it. The results are presented in Table 5. A larger $\lambda$ in the straight road indicates the extent to which the RMS delay spread variation is greater.

3.4. RMS Angular Spread. The RMS angular spread is calculated as

$$
\mathrm{AS}=\sqrt{-2 \ln \left(\left|\frac{\left.\sum_{n=1}^{N} e^{\left(j \theta_{n}\right)}\right) P_{n}}{\sum_{n=1}^{N} P_{n}}\right|\right)},
$$

where $P_{n}$ and $\theta_{n}$ denote the power and angle (azimuth/elevation angle of arrival/departure) of the $n$-th ray, respectively, and $N$ is the number of rays. 


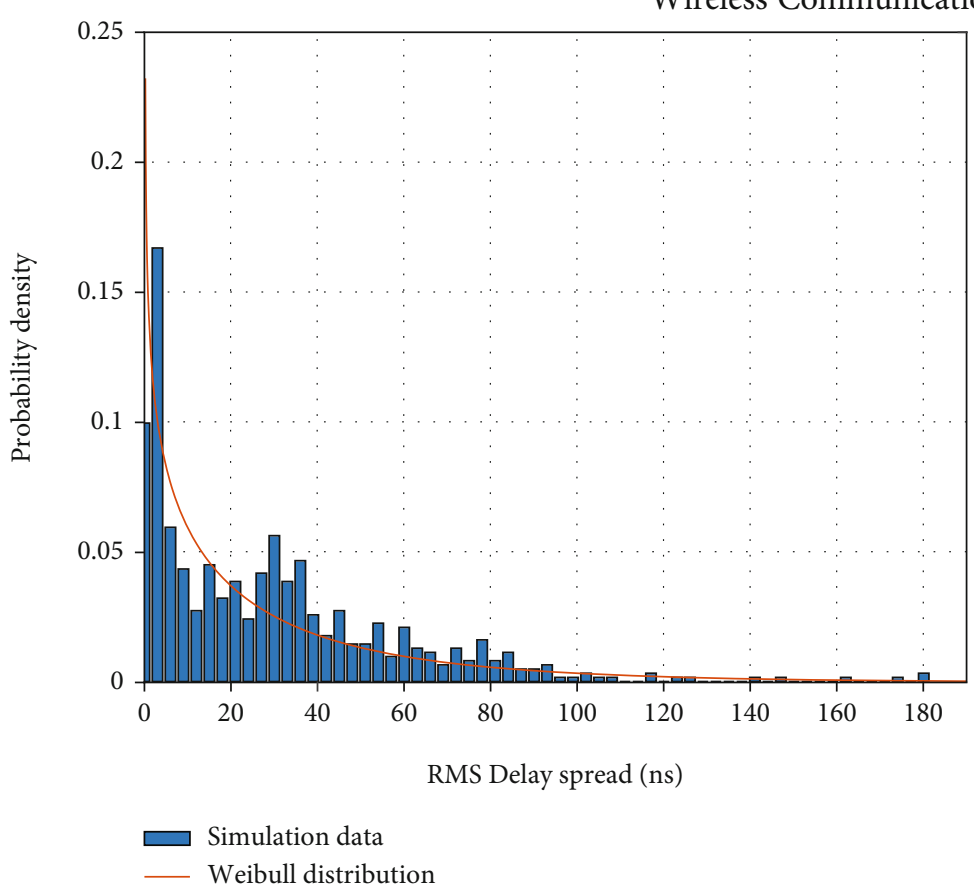

(a)

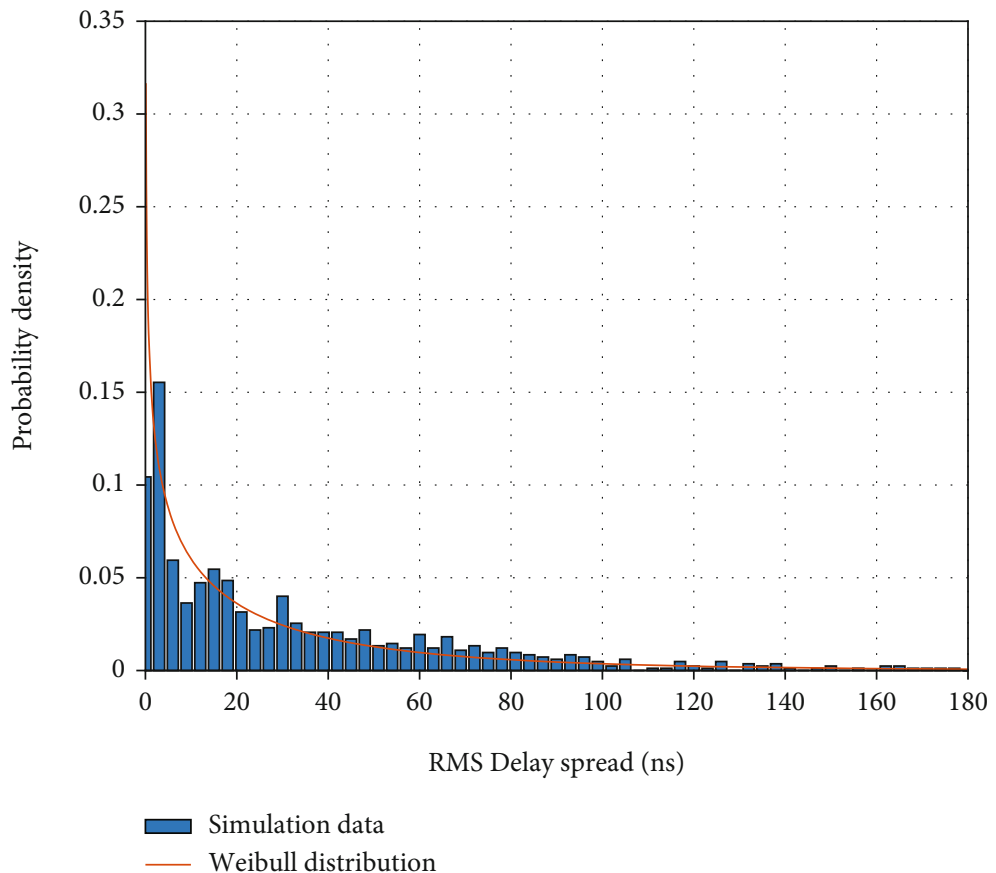

(b)

Figure 13: PDFs of RMS delay spread: (a) straight road; (b) intersection.

TABLE 5: Parameters in RMS delay spread.

\begin{tabular}{lcc}
\hline Scenario & Straight road & Intersection \\
\hline$\lambda$ & 27.55 & 26.16 \\
$k$ & 0.80 & 0.74 \\
\hline
\end{tabular}

In the $\mathrm{V} 2 \mathrm{~V}$ channel, the angle of arrival and the angle of departure are equivalent. Therefore, only the RMS angular spread of departure is discussed. Figure 14 shows the CDFs of the Azimuth Spread of Departure (ASD) and Elevation Spread of Departure (ESD) in the straight road and the intersection.

ESDs are almost $0^{\circ}$ which indicate that the directions of the rays are almost parallel to the ground. The MPCs from the top of the buildings on both sides experience greater attenuation. The LOS path and the reflection paths from the buildings at the same height as the car contribute the main energy.

The median values of ASDs are $8.55^{\circ}$ in the straight road and $8.68^{\circ}$ in the intersection. $90 \%$ of ASDs are less than $32^{\circ}$ in 


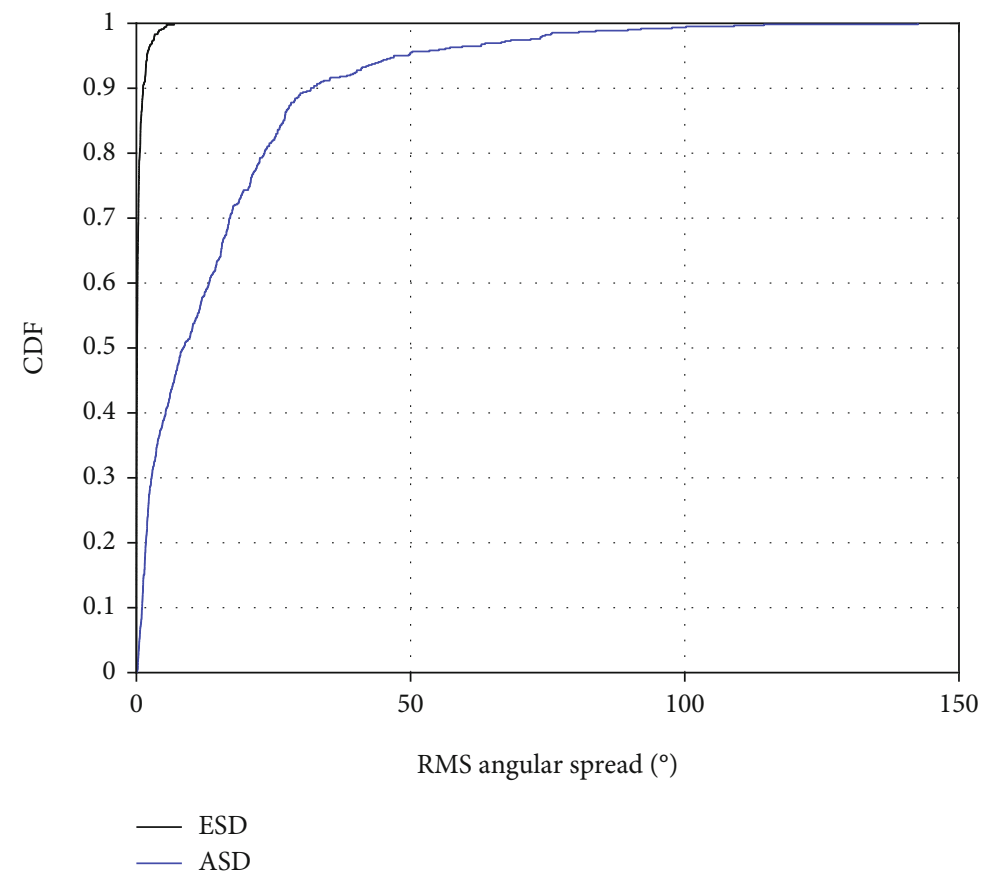

(a)

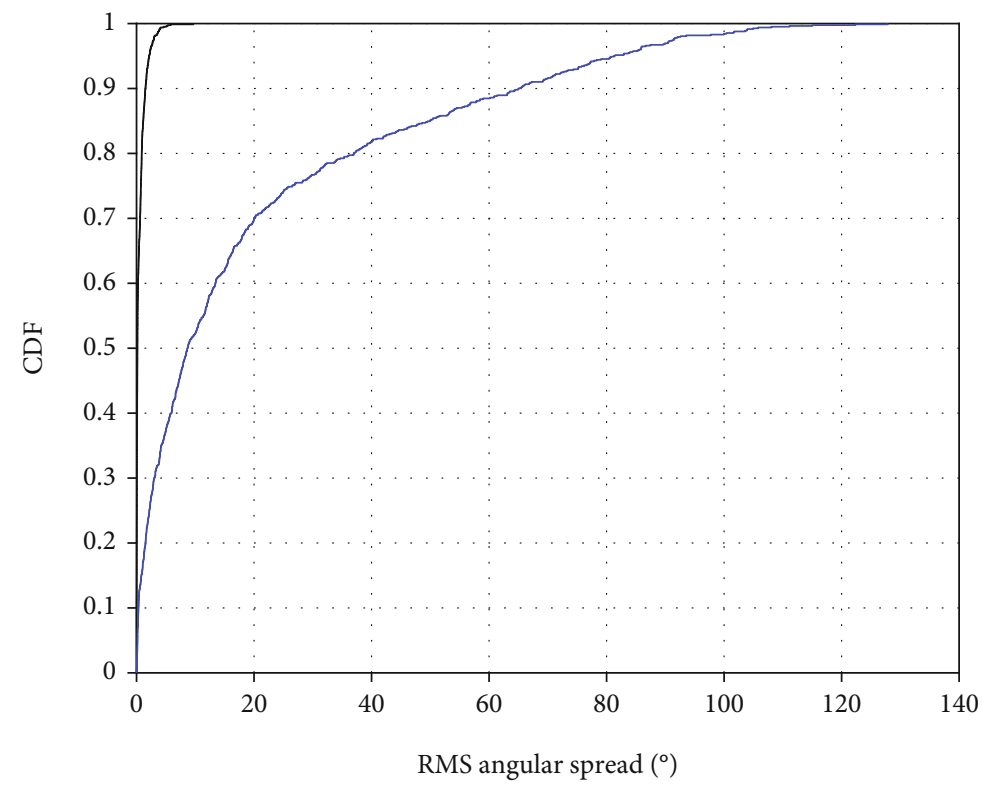

- ESD

ASD

(b)

FIGURE 14: CDFs of RMS angular spread: (a) straight road; (b) intersection.

the straight road and $56^{\circ}$ in the intersection. The ASDs in the intersection are larger than those in the straight road. The objects involved in the radio wave propagation in the straight road are arranged along both sides of the road, resulting in a relatively weak reflection and scattering paths.

The RMS angular spreads of the azimuth angle are larger than those of the elevation angle, illustrating that the low correlation and higher multiple-input multiple-output (MIMO) gain can be improved when the antenna arrays are placed horizontally at the Tx locations.

\section{Conclusion}

Based on RT simulation technology, the V2V channel characteristics at the $5.9 \mathrm{GHz}$ band are investigated. The two $\mathrm{V} 2 \mathrm{~V}$ scenarios, urban straight road and intersection, 
including road models, vehicle models, building models, and tree models, are established. RT simulation results show that the LOS probability nearly linearly decreases with the Tx-Rx distance, and the shadowing loss caused by obstructing vehicles can exceed $20 \mathrm{~dB}$ even in the short Tx-Rx distance. The shadowing loss can be well characterized by the multimodal Gaussian distribution. Thus, the path loss models in the LOS and NLOS conditions are obtained. Furthermore, the distributions of the RMS delay spread in the two scenarios are obtained and are modelled by the Weibull distribution. Finally, the deployment of antenna arrays is discussed based on the statistics distribution of the angular spread.

In the past, the shadowing effect caused by obstructing vehicles is usually modelled as one Gaussian-correlated variable. However, different blocking levels, as we have observed, have different effects on shadow fading, which have largely been ignored. More importantly, we propose a multimodal Gaussian distribution to describe the shadowing loss, and the division of the multimode depends on the difference of multipath components caused by different blocking levels.

In the future work, based on RT simulation, we will carry out the research on $\mathrm{V} 2 \mathrm{~V}$ channel characteristics of specific scenarios and measurement campaigns to verify the RT simulation results and make the results more convincing.

\section{Data Availability}

The data used to support the findings of this study are available from the corresponding author upon request.

\section{Conflicts of Interest}

The authors declare that there is no conflict of interest regarding the publication of this paper.

\section{Acknowledgments}

This work was supported by the Key-Area Research and Development Program of Guangdong Province under Grant 2019B010157002, the fund of the National Defense Science and Technology Key Laboratory for Equipment PreResearch (No: 6142217200404), the NSFC under Grant 61725101 and Grant U1834210, the Royal Society Newton Advanced Fellowship under Grant NA191006, the Natural Science Foundation of China under Grant 61961130391, the State Key Laboratory of Rail Traffic Control and Safety under Grants RCS2020ZT010 and RCS2019ZZ007, and the Open Research Fund from Shenzhen Research Institute of Big Data, under Grant No. 2019 ORF01006.

\section{References}

[1] T. Abbas, J. Nuckelt, T. Kurner, T. Zemen, C. F. Mecklenbrauker, and F. Tufvesson, "Simulation and measurement-based vehicle-to-vehicle channel characterization: accuracy and constraint analysis," IEEE Transactions on Antennas and Propagation, vol. 63, no. 7, pp. 3208-3218, 2015.

[2] Q. Wang, D. W. Matolak, and B. Ai, "Shadowing characterization for 5-GHz vehicle-to-vehicle channels," IEEE Transac- tions on Vehicular Technology, vol. 67, no. 3, pp. 1855-1866, 2018.

[3] J. Kunisch and J. Pamp, "Wideband car-to-car radio channel measurements and model at $5.9 \mathrm{GHz}$," in 2008 IEEE 68th Vehicular Technology Conference, pp. 1-5, Calgary, AB, Canada, 2008.

[4] L. Cheng, B. Henty, D. Stancil, F. Bai, and P. Mudalige, "Mobile vehicle to-vehicle narrow-band channel measurement and characterization of the $5.9 \mathrm{GHz}$ dedicated short range communication (DSRC) frequency band," IEEE Journal on Selected Areas in Communications, vol. 25, no. 8, pp. 15011516, 2007.

[5] I. Sen and D. W. Matolak, "Vehicle-vehicle channel models for the 5-GHz band," IEEE Transactions on Intelligent Transportation Systems, vol. 9, no. 2, pp. 235-245, 2008.

[6] O. Renaudin, V. Kolmonen, P. Vainikainen, and C. Oestges, "Wideband MIMO car-to-car radio channel measurements at $5.3 \mathrm{GHz}$," in 2008 IEEE 68th Vehicular Technology Conference, pp. 1-5, Calgary, AB, Canada, 2008.

[7] A. Paier, J. Karedal, N. Czink et al., "Characterization of vehicle-to-vehicle radio channels from measurements at 5.2 GHz," Wireless Personal Communications, vol. 50, no. 1, pp. 19-32, 2009.

[8] G. Acosta-Marum and M. Ingram, "Six time- and frequencyselective empirical channel models for vehicular wireless LANs," IEEE Vehicular Technology Magazine, vol. 2, no. 4, pp. 4-11, 2007.

[9] J. Karedal, F. Tufvesson, T. Abbas et al., "Radio channel measurements at street intersections for vehicle-to-vehicle applications," in 2010 IEEE 71st Vehicular Technology Conference, pp. 1-5, Taipei, Taiwan, 2010.

[10] C. Schneider, M. Kaske, G. Sommerkorn et al., "Directional analysis of vehicle-to-vehicle propagation channels," in 2011 IEEE 73rd Vehicular Technology Conference (VTC Spring), pp. 1-5, Budapest, Hungary, 2011.

[11] I. Tan, W. Tang, K. Laberteaux, and A. Bahai, "Measurement and analysis of wireless channel impairments in DSRC vehicular communications," in 2008 IEEE International Conference on Communications, pp. 4882-4888, Beijing, China, 2008.

[12] J. Nuckelt, T. Abbas, F. Tufvesson, C. Mecklenbrauker, L. Bernado, and T. Kurner, "Comparison of ray tracing and channel-sounder measurements for vehicular communications," in 2013 IEEE 77th Vehicular Technology Conference (VTC Spring), pp. 1-5, Dresden, Germany, 2013.

[13] D. W. Matolak, "V2V communication channels: state of knowledge, new results, and what's next," in International Workshop on Communication Technologies for Vehicles, pp. 1-22, Villeneuve d'Ascq, France, 2013.

[14] T. Abbas, K. Sjöberg, J. Karedal, and F. Tufvesson, "A measurement based shadow fading model for vehicle-to-vehicle network simulations," International Journal of Antennas and Propagation, vol. 2015, Article ID 190607, 12 pages, 2015.

[15] P. Liu, D. W. Matolak, B. Ai, and R. Sun, "Path loss modeling for vehicle-to-vehicle communication on a slope," IEEE Transactions on Vehicular Technology, vol. 63, no. 6, pp. 2954-2958, 2014.

[16] C. A. Gutiérrez, J. J. Jaime-Rodríguez, J. M. Luna-Rivera, D. U. Campos-Delgado, and J. Vázquez Castillo, "Modeling of nonWSSUS double-Rayleigh fading channels for vehicular communications," Wireless Communications and Mobile Computing, vol. 2017, Article ID 6394653, 15 pages, 2017. 
[17] R. Meireles, M. Boban, P. Steenkiste, O. Tonguz, and J. Barros, "Experimental study on the impact of vehicular obstructions in VANETs," in 2010 IEEE Vehicular Networking Conference, pp. 338-345, Jersey City, NJ, USA, 2010.

[18] J. Zhao, Y. Ji, and Y. Li, "Analysis on characteristics of road traffic flow in Tianjin," Environmental Science Research, vol. 32, no. 3, pp. 399-405, 2019.

[19] P. Zhang and X. Li, "Architectural types and spatial distribution characteristics of southern district of Qingdao," Journal of Ecology, vol. 33, no. 7, pp. 1882-1887, 2014.

[20] K. Guan, Z. Zhong, B. Ai, and T. Kürner, "Deterministic propagation modeling for the realistic high-speed railway environment," in 2013 IEEE 77th Vehicular Technology Conference (VTC Spring), pp. 1-5, Dresden, Germany, 2013.

[21] S. Priebe and T. Kürner, "Stochastic modeling of THz indoor radio channels," IEEE Transactions on Wireless Communications, vol. 12, no. 9, pp. 4445-4455, 2013.

[22] D. He, B. Ai, K. Guan, L. Wang, Z. Zhong, and T. Kürner, “The design and applications of high-performance ray-tracing simulation platform for $5 \mathrm{G}$ and beyond wireless communications: a tutorial," IEEE Communications Surveys \& Tutorials, vol. 21, no. 1, pp. 10-27, 2019.

[23] D. He, K. Guan, A. Fricke et al., "Stochastic channel modeling for kiosk applications in the terahertz band," IEEE Transactions on Terahertz Science and Technology, vol. 7, no. 5, pp. 502-513, 2017.

[24] S. Ju, S. H. A. Shah, M. A. Javed et al., "Scattering mechanisms and modeling for terahertz wireless communications," in ICC 2019 - 2019 IEEE International Conference on Communications (ICC), pp. 1-7, Shanghai, China, 2019.

[25] ITU-R. R. P.1411, "Propagation data and prediction methods for the planning of short-range outdoor radio communication systems and radio local area networks in the frequency range $300 \mathrm{MHz}$ and $100 \mathrm{GHz}$," ITU, 2012.

[26] J. Lee, M. D. Kim, H. K. Chung, and J. Kim, "NLOS path loss model for low-height antenna links in high-rise urban street grid environments," International Journal of Antennas and Propagation, vol. 2015, Article ID 651438, 9 pages, 2015.

[27] A. F. Molisch, F. Tufvesson, J. Karedal, and C. Mecklenbrauker, "A survey on vehicle-to-vehicle propagation channels," IEEE Wireless Communications, vol. 16, no. 6, pp. 12-22, 2009.

[28] J. Karedal, N. Czink, A. Paier, F. Tufvesson, and A. F. Molisch, "Path loss modeling for vehicle-to-vehicle communications," IEEE Transactions on Vehicular Technology, vol. 60, no. 1, pp. 323-328, 2011.

[29] H. Fernández, L. Rubio, J. Reig, V. M. Rodrigo-Peñarrocha, and A. Valero, "Path loss modeling for vehicular system performance and communication protocols evaluation," Mobile Networks and Applications, vol. 18, no. 6, pp. 755-765, 2013.

[30] Y. Li, B. Ai, X. Cheng, S. Lin, and Z. Zhong, "A TDL based non-WSSUS vehicle-to-vehicle channel model," International Journal of Antennas and Propagation, vol. 2013, Article ID 103461, 8 pages, 2013.

[31] T. Abbas, J. Karedal, F. Tufvesson, A. Paier, L. Bernado, and A. F. Molisch, "Directional analysis of vehicle-to-vehicle propagation channels," in 2011 IEEE 73rd Vehicular Technology Conference (VTC Spring), pp. 1-5, Budapest, Hungary, 2011.

[32] J. D. Parsons, The Mobile Radio Propagation Channel, Wiley, Hoboken, NJ, USA, 2nd edition, 2000.
[33] D. W. Matolak, I. Sen, W. Xiong, and N. T. Yaskoff, " $5 \mathrm{GHz}$ wireless channel characterization for vehicle to vehicle communications," in MILCOM 2005 - 2005 IEEE Military Communications Conference, pp. 3016-3022, Atlantic City, NJ, USA, 2005.

[34] A. F. Molisch, F. Tufvesson, J. Karedal, and C. Mecklenbrauker, "Propagation aspects of vehicle-to-vehicle communications - an overview," in 2009 IEEE Radio and Wireless Symposium, pp. 179-182, San Diego, CA, USA, 2009. 